\title{
Percutaneous External Locking Plate Fixation Versus Open Reduction with Internal Fixation for the Protection of Supraclavicular Nerve in the Treatment of Clavicle Fracture
}

\author{
Abulaiti Abula \\ the First Affiliated Hospital of Xinjiang Medical University \\ Yanshi Liu \\ the First Affiliated Hospital of Xinjiang Medical University \\ Kai Liu \\ the First Affiliated Hospital of Xinjiang Medical University \\ Feiyu Cai \\ the First Affiliated Hospital of Xinjiang Medical University \\ Alimujiang Abulaiti \\ the First Affiliated Hospital of Xinjiang Medical University \\ Xiayimaierdan Maimaiti \\ the First Affiliated Hospital of Xinjiang Medical University \\ Peng Ren \\ the First Affiliated Hospital of Xinjiang Medical University \\ Aihemaitijiang Yusufu ( $\nabla$ ahmatjang@163.com ) \\ the First Affiliated Hospital of Xinjiang Medical University
}

\section{Research Article}

Keywords: Clavicle fracture, Open reduction and internal fixation, Supraclavicular nerve injury

Posted Date: October 25th, 2021

DOI: https://doi.org/10.21203/rs.3.rs-997593/v1

License: (c) (i) This work is licensed under a Creative Commons Attribution 4.0 International License. Read Full License 


\section{Abstract}

Background: The purpose of this study was to determine the differences in the clinical effectiveness of supraclavicular nerve protection, if any, between percutaneous external locking plate fixation and open reduction with internal fixation (ORIF) in the treatment of clavicle fracture.

Methods: A total of 27 patients who suffered clavicle fracture and underwent treatment of percutaneous external locking plate fixation or ORIF in our department, from January 2015 to January 2020, were retrospectively collected. There were 19 males and 8 females with a mean age of 42 years (range 21 to 57 years). Supraclavicular nerves were protected in all patients during the operation procedures. Among them, 17 patients were treated by percutaneous external locking plate fixation, while the other 10 patients were treated by ORIF. The paresthesia in the affected shoulder and the superior lateral thoracic area after surgery was collected and analyzed. Additionally, the satisfactory rate after the fixation removal was used to evaluate the clinical effectiveness.

Results: All 27 patients were successfully followed with a mean duration of 1.3 years ( 1 to 5.8 years), and all fractures achieved bone union finally. There were significant differences in paresthesia in the affected shoulder area and the superior lateral thoracic area between the two groups after surgery $(P<0.05)$. The satisfactory rate in the percutaneous external locking plate fixation group was higher than that in the ORIF group after the fixation removal $(P<0.05)$.

Conclusions: Clavicle fracture treated by percutaneous external locking plate fixation has benefits in the protection of supraclavicular nerve, reducing the incidence of postoperative paresthesia in the affected shoulder and superior lateral thoracic area.

\section{Background}

The clavicular epithelial nerve comes from the superficial branches of the anterior ramus of the cervical plexus, originating from the 3rd and 4th cervical nerves. It goes from the midpoint of the posterior edge of the sternocleidomastoid muscle shallowly out of the skin, runs on the deep surface of the platysma to near the clavicle, and penetrates subcutaneously. It is divided into three branches, namely medial, middle and lateral branch, and distributes in the shoulder area and the superior lateral thoracic area across the clavicle.

Clavicle fractures are prone to occur in one-third of the medial-lateral clavicle [1-7], which belongs to the area where the supraclavicular nerves are distributed. Clavicle fractures are mainly treated with ORIF, in which the supraclavicular nerve is easily damaged, and the incidence of paresthesia such as numbness and discomfort in the affected shoulder and superior lateral thoracic area after surgery is as high as about $30 \%[1,3,4,8-10]$. Previous clinical studies have found that the main cause of paresthesia in the affected shoulder area and superior lateral thoracic area under ORIF is the injury to the supraclavicular nerve during the operation $[2,5,11]$. 
In the present study, 27 patients were treated with open reduction fixation with reservation of the clavicular epithelial nerve. We tried to protect the supraclavicular nerves during operation procedures to improve postoperative skin numbness. The purpose of this study was to determine the differences in the clinical effectiveness of supraclavicular nerve protection, if any, between percutaneous external locking plate fixation and open reduction with internal fixation (ORIF).

\section{Methods}

In this study, a total of 27 patients who suffered clavicle fracture and underwent treatment of percutaneous external locking plate fixation or ORIF in our department, from January 2015 to January 2020, were retrospectively collected. There were 19 males and 8 females with a mean age of 42 years (range 21 to 57 years). All patients suffered unilaterally closed clavicle fracture, and the time from fracture to operation was less than 2 weeks. Clavicle fractures with other parts combined fractures, old and pathological fractures, combined acromioclavicular joint and sternoclavicular joint dislocation, and mental retardation were excluded. The types of clavicle fractures included transverse fracture in 5 cases, oblique fracture in 8 cases, and comminuted fracture in 14 cases. According to the different treatments, the patients were divided into two groups: the percutaneous external locking plate fixation group and the ORIF group. Among them, 17 patients ( 15 males, 2 females) received percutaneous external locking plate fixation, including 2 cases of transverse fractures, 5 cases of oblique fractures, and 10 cases of comminuted fractures. The other 10 patients (4 males, 6 females) underwent ORIF, including 3 cases of transverse fractures, 3 cases of oblique fractures, and 4 cases of comminuted fractures.

\section{Surgical technique}

\section{Percutaneous external locking plate fixation group}

The surgical procedure was as follows: Firstly, patient with a supine position and tilted the head to the healthy side underwent general anesthesia or brachial plexus block anesthesia, color doppler ultrasound was used to locate the supraclavicular nerve and mark the surface of the skin (as shown in Figure 1) at the same time. Secondly, performing a conventional disinfection drape. Thirdly, making a $2 \sim 3 \mathrm{~cm}$ surgical incision centering on the fracture of the clavicle, cutting the skin and separate the subcutaneous tissue, then performing blunt dissection in the subcutaneous fat layer carefully with vascular forceps to reach the deep fascia layer of the platysma muscle; probe and protect the branch of the supraclavicular nerve that perpendicular to the clavicle on the surface of the clavicle; the clavicular epithelial nerves are moderately dissociated and protected, and should not be excessively stretched. Fourthly, reduce the fracture, placing the clavicle locking plate $1 \sim 1.5 \mathrm{~cm}$ away from the skin, and fixing both ends of the fracture with two locking screws, the length of which is generally about $26 \mathrm{~mm}$. Because the branches of the clavicular epithelial nerve were localized and marked by ultrasound preoperatively, the clavicular epithelial nerve branches that are not in the incision could be avoided during percutaneous screw fixation to avoid damage to the clavicular epithelial nerve branches. After the operation, a forearm sling was used 
to protect the affected upper limb for 3 weeks, and postoperative antibiotics were routinely used for $1 \sim 3$ days.

\section{ORIF group}

The surgical procedure was as follows: Firstly, patient with a supine position and tilted the head to the healthy side underwent general anesthesia or brachial plexus block anesthesia, color doppler ultrasound was used to locate the supraclavicular nerve and mark the surface of the skin (as shown in Figure 2) at the same time. Secondly, performing a conventional disinfection drape. Thirding, making an $8 \sim 10 \mathrm{~cm}$ surgical incision centering on the fracture of the clavicle, cut the skin and separate the subcutaneous tissue, then perform blunt dissection in the subcutaneous fat layer carefully with vascular forceps to reach the deep fascia layer of the platysma muscle according to the preoperative ultrasound position mark, and 2 or 3 branches of the supraclavicular nerve can be found on the surface of the clavicle that almost perpendicular to the clavicle; reserve a small amount of fatty soft tissue around the supraclavicular nerve to avoid excessive nakedness of the supraclavicular nerve; separate the platysma muscle to release and protect the epithelial nerve depending on the circumstances, and the clavicular epithelial nerves should not be excessively stretched. Fourthly, stripping part of the periosteum and revealing the broken end of the clavicle fracture, then reducing the broken end of the fracture under direct vision, with care to protect the blood supply of the fracture end during this process. For patients with comminuted clavicle fractures, the bone fragments could be restored and fixed with Kirschner pins first, and then the broken ends of the fractures were reduced. After avoiding the supraclavicular nerve, an anatomical plate was placed on the surface of the clavicle, and the fractured end was internally fixed with screws. Fifthly, flushing the field, suturing the incision, and using a forearm sling to protect the affected upper limb for 3 weeks after the operation. Postoperative antibiotics were routinely used for 1 3 days. Special attention should be taken not to suture or ligate the clavicular epithelial nerve in the line knot when suturing the surgical incision, indwelling skin for drainage.

\section{Results}

All the 27 patients were successfully followed with a mean duration of 1.3 years ( 1 to 5.8 years), and all fractures achieved bone union finally. There were significant differences in paresthesia in the affected shoulder area and the superior lateral thoracic area between the two groups after surgery $(P<0.05)$. The satisfactory rate in the percutaneous external locking plate fixation group was higher than that in the ORIF group after the fixation removal $(P<0.05)$. (Table 1$)$

Two cases of the percutaneous external locking plate fixation group developed postoperative redness and swelling around the external screw (Figure 1d), and were successfully managed by anti-inflammatory and dressing change. Two patients developed hyposensitization in the affected shoulder and upper chest area and were successfully treated by oral Methycobal Capsules.

In the ORIF group, 2 patients showed hypoesthesia in the affected shoulder and upper thoracic area after the operation, which returned to normal after 1 year. Another 3 patients had supraclavicular nerve injury 
during the removal of internal fixation one year later. Further follow-up of these patients revealed sensory dysfunction, including pricking, tactile hyperalgesia, and local numbness in the operation area as well as the affected neck and shoulder.

\section{Typical case}

A 32-year-old male suffered supraclavicular nerve injury during the second incision for removing the internal fixation, symptoms include night pain, dull pain, and prickling accompanied by itching. The pain radiated to the head, neck, shoulders, or back, accompanied by neck stiffness and spasm and paralysis of the muscles, followed by other symptoms such as anxiety, and hyperesthesia or hypoesthesia may appear in the nerve distribution area. After continuous follow-up (outpatient follow-up every 3-6 months), the patient gradually improved about 3.8 years later. Five years later, normal sleeping and no pain in the neck and shoulder area were obtained, and sensory dysfunction in the affected shoulder area and the superior lateral thoracic area returned to normal, but there still had dull pain at the $1 / 3$ of the mediallateral of the clavicle. As shown in Figure3, the hypoesthesia area after the 1-year postoperative follow-up is $15.5 \mathrm{~cm} \times 13.5 \mathrm{~cm}$, while showed $9 \mathrm{~cm} \times 5 \mathrm{~cm}$ after the 5 -year postoperative follow-up. (Figure 3 )

\section{Discussion}

\section{Mechanism of clavicle epithelium injury}

The supraclavicular nerves come from the superficial branch of the anterior branch of the cervical plexus. The nerve originating from the $\mathrm{C} 3$ and $\mathrm{C} 4$ nerves is the cutaneous nerve, which exists from the anterior of scalenus medius at $1 / 2$ of the posterior margin of the sternocleidomastoid muscle and is immediately divided into three branches, namely the medial, middle, and lateral branches. Then it enters the posterior cervical triangle and lies between the deep and superficial fascia, and pass through the superficial layer of cervical fascia and the lower part of the platysma muscle in turn, and crosses distally over the front of the clavicle, dominating the sense of shoulders, upper chest, and lower neck. The most predisposed site of clavicle fractures is distributed at $1 / 3$ of the medial-lateral of the clavicle $[8,10,12-14]$, and it is relatively easy to damage the clavicular epithelial nerve during fracture or open reduction and internal fixation $[3,4,6,11]$. In the past, orthopedic surgeons often ignored the protection of supraclavicular nerves. Clavicular epithelial nerve injury leads to partial or complete loss of skin sensory function in the corresponding areas such as the shoulder and upper chest, and increases the incidence of traumatic or scar neuroma, which causes "electric shock-like" prickling and affects sleep at night.

Although the skin paraesthesia of the affected side appeared after surgery, which did not affect the motor function of the upper limbs, the patient complained of numbness, prickling, itching, burning sensation, and other discomforts in the affected shoulder area and superior lateral thoracic area after surgery. The main manifestations were discomfort such as neck and shoulder pain and soreness $[3,4,15]$ : Pain: Acute attack, which can be a dull pain, swelling pain, or prickling that can radiate to the head and neck or shoulder-back. Restriction of movement: Restriction of movement of the neck, accompanied by neck 
stiffness and spasm and paralysis of the muscles under its control. Paresthesia: Hyperesthesia or hypoesthesia may occur in the nerve distribution area, which affects the daily life of the patient, thereby reducing the patient's quality of life and satisfaction with the surgical effect. Therefore, iatrogenic clavicular epithelial nerve injury should be reduced during the operation, to reduce postoperative paresthesia and improve patient satisfaction.

\section{Advantages and disadvantages of percutaneous external locking plate fixation}

It has been reported that minimally invasive surgery for patients with clavicle fractures can reduce the surgical incision of the skin and improve the postoperative aesthetics of the surgical incision, but it cannot avoid the injury of supraclavicular nerve during the operation [15-19]. O'Neill et al. [20] reported that longitudinal skin incisions were placed with clavicle hook plates to avoid clavicular epithelial nerve injury. For the 10 cases with ORIF in the present study, although the supraclavicular nerve was effectively protected during the first phase of the operation, it could still exhibit symptoms of nerve irritation due to the friction stimulation between the steel plate and supraclavicular nerve. In this group, three patients suffered iatrogenic clavicular epithelial nerve injury when the internal fixation was moved, and suffered sensory dysfunction in the affected shoulder and superior lateral thoracic areas. In the percutaneous external locking plate fixation group, the incidence of paresthesia in the affected shoulder and the superior lateral thoracic area was lower than the ORIF group within 1 year after the operation. Two of the patients developed hypoesthesia but returned to normal 1 year later.

The advantage of percutaneous external locking plate fixation was the reduced possibility of injury to the supraclavicular nerve in the first stage and the second stage [20-23]. However, there are also shortcomings: postoperative redness and swelling around the external screw occurred in 2 cases, and were successfully treated by anti-inflammatory and dressing change. Long-term pin site care and the cumbersome appearance lead to psychological repellence in a small number of patients, requiring meticulous care and regular follow-up.

In summary, our experiences manifested percutaneous external locking plate fixation can effectively avoid further damage to the local blood supply, and external elastic fixation can stimulate callus regeneration. In addition, the possibility of clavicular epithelial nerve injury can be reduced by small longitudinal incisions with nail holes. Furthermore, percutaneous external locking plate fixation can effectively reduce the nerve stimulation caused by the friction between the plate and the supraclavicular nerve. Particularly, there is no need to hospitalization and re-operation for the removal of internal fixation, which reduces the patient's medical expenditure and avoids the possibility of the supraclavicular nerve injury during the fixation removal.

\section{Conclusion}


Clavicle fracture treated by percutaneous external locking plate fixation has benefits in the protection of the supraclavicular nerve, reducing the incidence of postoperative paresthesia in the affected shoulder and superior lateral thoracic area.

\section{Abbreviations}

ORIF: open reduction and internal fixation

\section{Declarations}

\section{Ethics approval and consent to participate}

All methods in this study were carried out in accordance with the Declaration of Helsinki. This study was approved by the Ethics Committee of The First Affiliated Hospital of Xinjiang Medical University. Written informed consent was obtained from all patients for their data to be recorded in our study.

\section{Consent for publication}

Informed consent was obtained from all patients for their data to be published in our study.

\section{Availability of data and materials}

The datasets generated and analysed during the current study are not publicly available due to limitations of ethical approval involving the patient data and anonymity but are available from the corresponding author on reasonable request.

\section{Competing interests}

The authors declare that they have no competing interests.

\section{Funding}

This study was funded by Natural Science Foundation of Xinjiang (No.2020D01C250) and National Natural Science Foundation of China (No. 82060401). The funding body was involved in the collection, analysis, and interpretation of data by supporting with salary for the time needed. They were not involved in the design or writing the manuscript.

\section{Authors' Contributions}

AA: Conducted the study. Collected, analyzed and interpreted the data. Wrote the manuscript.

YSL: Collected, interpreted and analyzed the data. Edited the manuscript.

KL: Created and statistical analyzed the data. Edited the manuscript. 
FYC: Provided software assistance. Edited the manuscript.

AA: Conducted the study and provided the data.

XM: Conducted the study and provided the data.

PR: Conducted the study and provided the data.

AY: Planned the project. Reviewed the manuscript.

Abulaiti Abula and Yanshi Liu contributed equally to this study.

All authors read and approved the final manuscript.

\section{Acknowledgements}

Not applicable

\section{References}

1. van der Meijden OA, Gaskill TR, Millett PJ. Treatment of clavicle fractures: current concepts review. J Shoulder Elbow Surg 2012;21:423-429

2. Woltz S, Krijnen P, Schipper IB. Mid-Term Patient Satisfaction and Residual Symptoms After Plate Fixation or Nonoperative Treatment for Displaced Midshaft Clavicular Fractures. J ORTHOP TRAUMA 2018;32:e435-e439

3. Sorensen AR, Hammeken LH, Qvist AH, Jensen SL, Ehlers LH. Operative treatment of displaced midshaft clavicular fractures is not cost-effective. J Shoulder Elbow Surg 2020;29:27-35

4. Ma X, Wang K, Ma J, Chen X, Han S. Operative treatments compared with nonoperative treatment of displaced midshaft clavicular fractures. J ORTHOP SCI 2020;25:310-314

5. Woltz S, Stegeman SA, Krijnen P, et al. Plate Fixation Compared with Nonoperative Treatment for Displaced Midshaft Clavicular Fractures: A Multicenter Randomized Controlled Trial. J BONE JOINT SURG AM 2017;99:106-112

6. Postacchini F, Gumina S, De Santis P, Albo F. Epidemiology of clavicle fractures. J Shoulder Elbow Surg 2002;11:452-456

7. Robinson CM. Fractures of the clavicle in the adult. Epidemiology and classification. J Bone Joint Surg $\mathrm{Br}$ 1998;80:476-484

8. Burnham JM, Kim DC, Kamineni S. Midshaft Clavicle Fractures: A Critical Review. ORTHOPEDICS 2016;39:e814-e821 
9. Frima $\mathrm{H}$, van Heijl M, Michelitsch C, et al. Clavicle fractures in adults; current concepts. Eur J Trauma Emerg Surg 2020;46:519-529

10. Woltz S, Krijnen P, Schipper IB. Plate Fixation Versus Nonoperative Treatment for Displaced Midshaft Clavicular Fractures: A Meta-Analysis of Randomized Controlled Trials. J BONE JOINT SURG AM 2017;99:1051-1057

11. Qvist AH, Vaesel MT, Jensen CM, Jensen SL. Plate fixation compared with nonoperative treatment of displaced midshaft clavicular fractures: a randomized clinical trial. BONE JOINT J 2018;100-B:1385-1391

12. Wiesel B, Nagda S, Mehta S, Churchill R. Management of Midshaft Clavicle Fractures in Adults. J Am Acad Orthop Surg 2018;26:e468-e476

13. Longo UG, Banerjee S, Barber J, et al. Conservative management versus open reduction and internal fixation for mid-shaft clavicle fractures in adults-the Clavicle Trial: study protocol for a multicentre randomized controlled trial. TRIALS 2011;12:57

14. Tutuhatunewa ED, Stevens M, Diercks RL. Clinical outcomes and predictors of patient satisfaction in displaced midshaft clavicle fractures in adults: Results from a retrospective multicentre study. INJURY 2017;48:2788-2792

15. Wenninger JJ, Dannenbaum JH, Branstetter JG, Arrington ED. Comparison of complication rates of intramedullary pin fixation versus plating of midshaft clavicle fractures in an active duty military population. J Surg Orthop Adv 2013;22:77-81

16. King PR, Lamberts RP. Management of clavicle shaft fractures with intramedullary devices: a narrative review. Expert Rev Med Devices 2020;17:807-815

17. Huang SG, Chen B, Lv D, et al. Evaluation of shoulder function in clavicular fracture patients after six surgical procedures based on a network meta-analysis. DISABIL REHABIL 2017;39:105-112

18. Khan LA, Bradnock TJ, Scott C, Robinson CM. Fractures of the clavicle. J BONE JOINT SURG AM 2009;91:447-460

19. Zehir S, Zehir R, Sahin E, Calbiyik M. Comparison of novel intramedullary nailing with mini-invasive plating in surgical fixation of displaced midshaft clavicle fractures. Arch Orthop Trauma Surg 2015;135:339-344

20. O'Neill K, Stutz C, Duvernay M, Schoenecker J. Supraclavicular nerve entrapment and clavicular fracture. J ORTHOP TRAUMA 2012;26:e63-e65

21. Mehta A, Birch R. Supraclavicular nerve injury: the neglected nerve? INJURY 1997;28:491-492 
22. Nathe T, Tseng $S$, Yoo B. The anatomy of the supraclavicular nerve during surgical approach to the clavicular shaft. Clin Orthop Relat Res 2011;469:890-894

23. Omokawa S, Tanaka Y, Miyauchi Y, Komei T, Takakura Y. Traction neuropathy of the supraclavicular nerve attributable to an osseous tunnel of the clavicle. Clin Orthop Relat Res 2005:238-240

\section{Tables}

Table 1 The incidence of paresthesia in the shoulder area and the superior lateral thoracic area of the affected side and the satisfaction rate of the surgical effect in the two groups after the removal of the fixed plate (\%)

\begin{tabular}{lccc}
\hline Group & Cases & $\begin{array}{l}\text { Incidence of paresthesia in the affected shoulder } \\
\text { area and the superior lateral thoracic area after } \\
\text { removing the fixation }\end{array}$ & Satisfaction rate \\
\hline $\begin{array}{l}\text { Percutaneous external } \\
\text { locking plate fixation }\end{array}$ & 17 & 0 & 82.35 \\
ORIF & 10 & $30 \%$ & $80 \%$ \\
$p$ value & & 0.04 & 0.02 \\
\hline
\end{tabular}

\section{Figures}




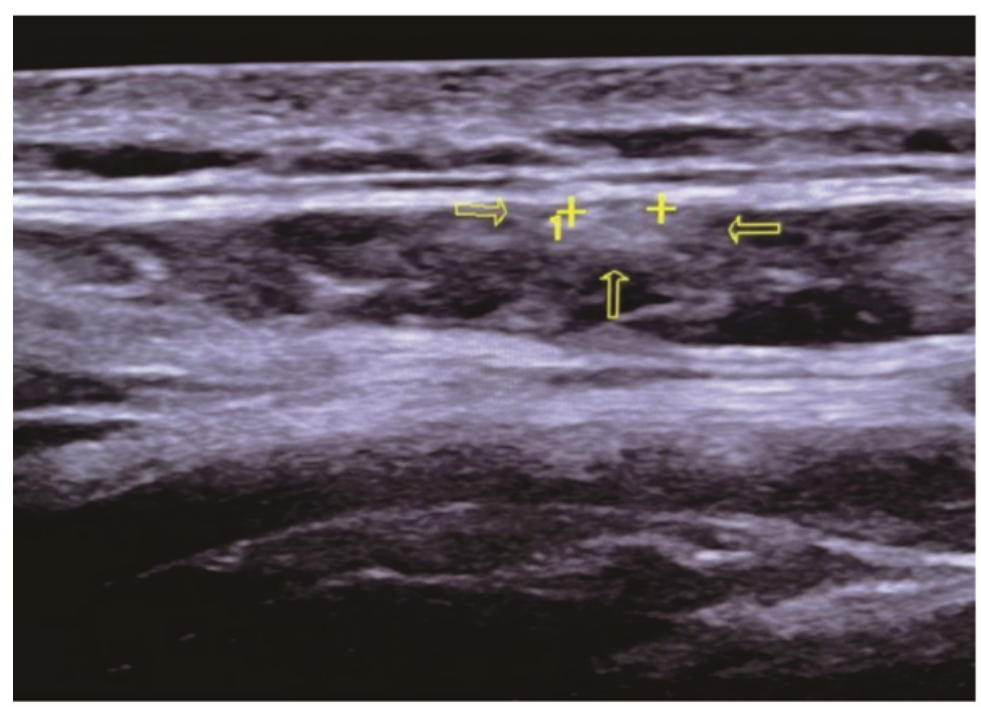

A

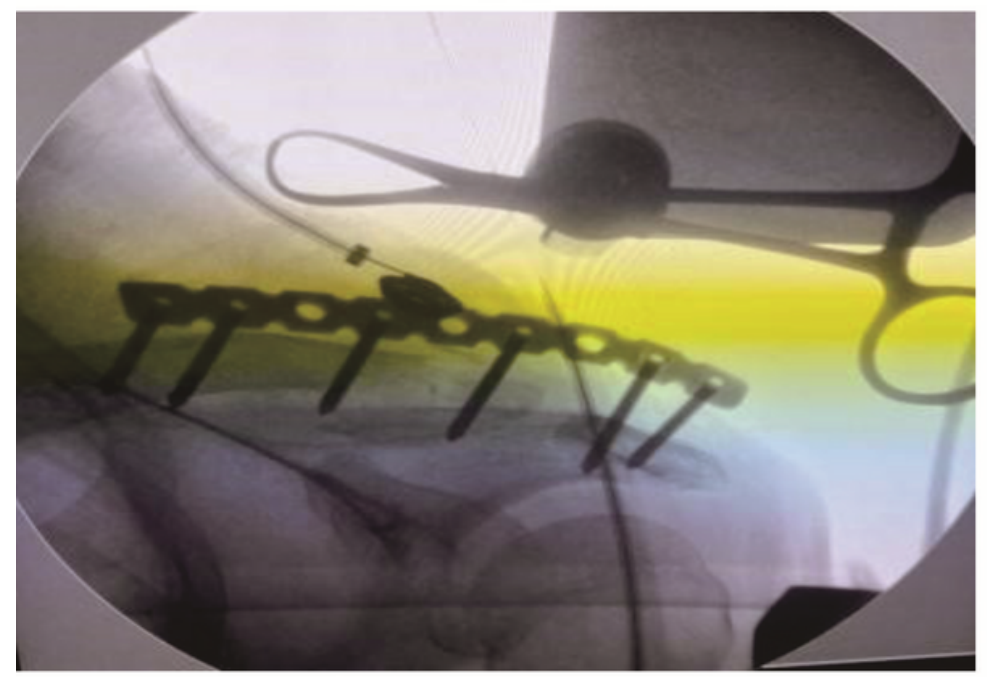

C

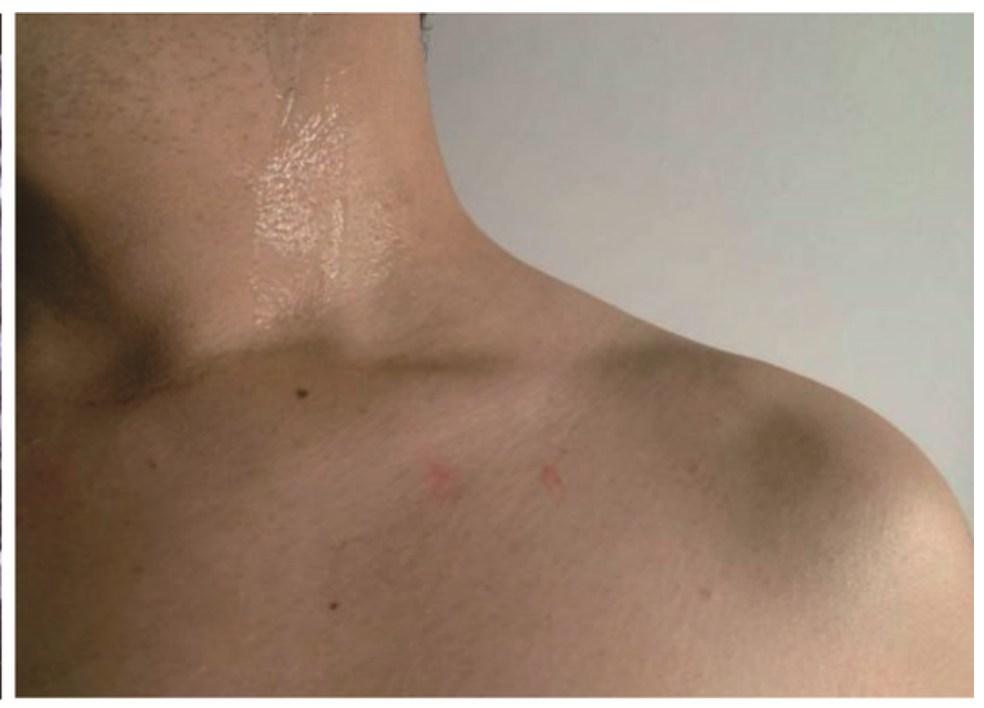

B

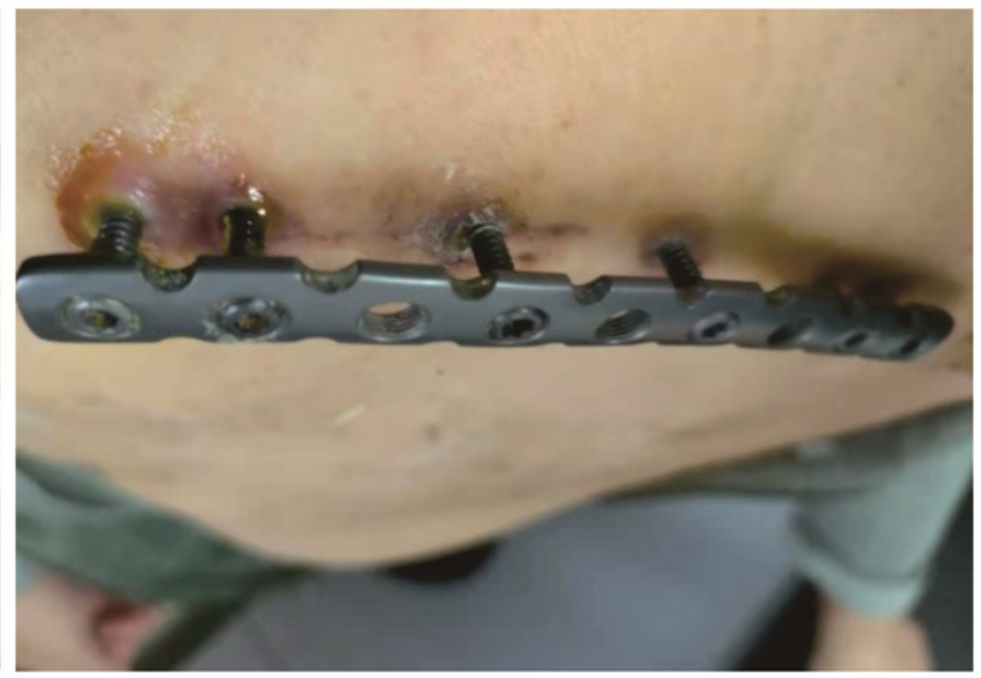

D

Figure 1

a Color doppler ultrasound was used to locate the supraclavicular nerve and mark the surface of the skin. b Surgical marks on the skin. c The radiograph of clavicle after external plate implantation. $d$ Appearance photo of shoulder after external plate implantation. 


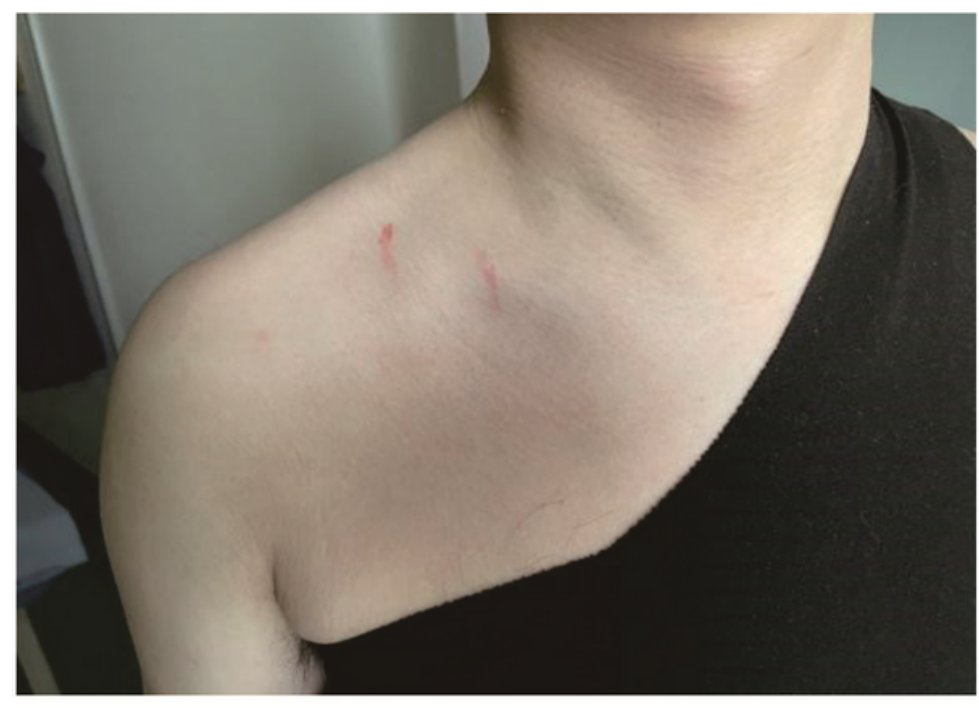

A

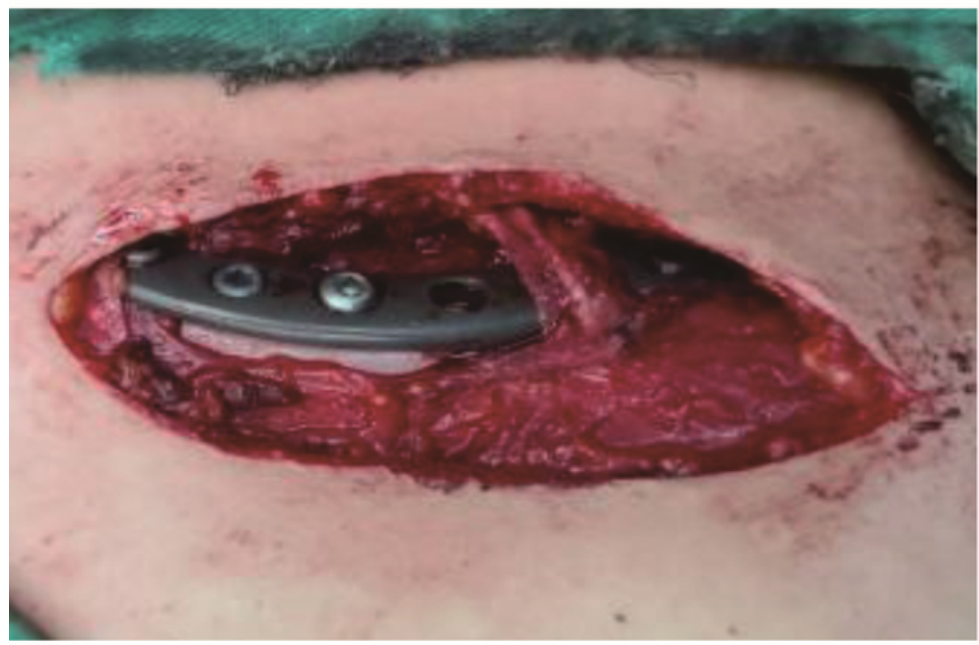

C

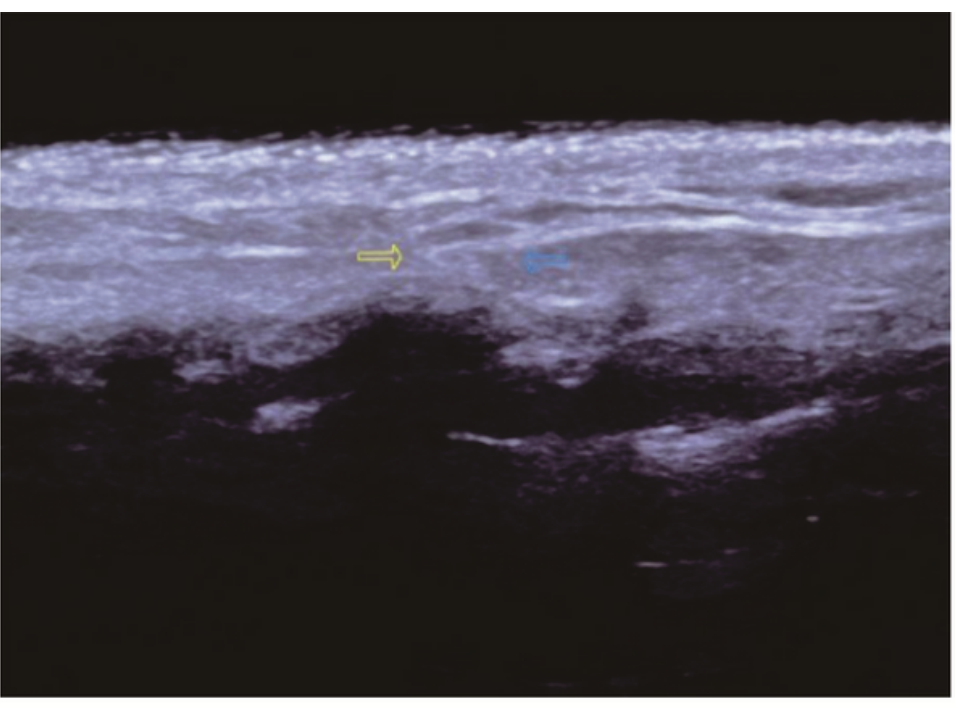

B

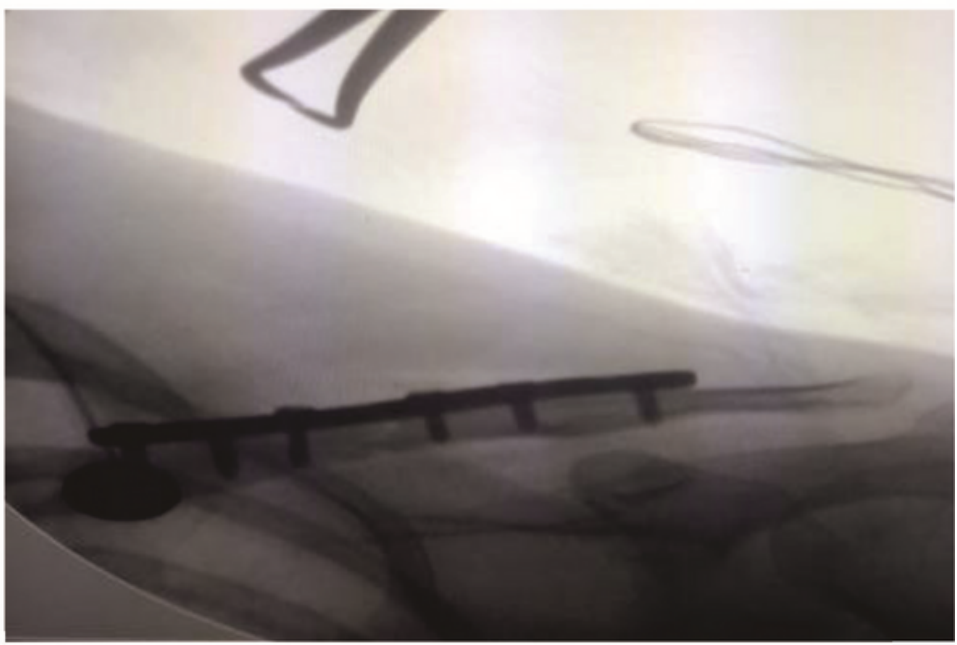

$\mathrm{D}$

Figure 2

a Surgical mark on the skin. b Color Doppler ultrasound was used to locate the supraclavicular nerve and mark the surface of the skin. c Appearance photo of shoulder after external plate implantation. $d$ The radiograph of clavicle after external plate implantation. 

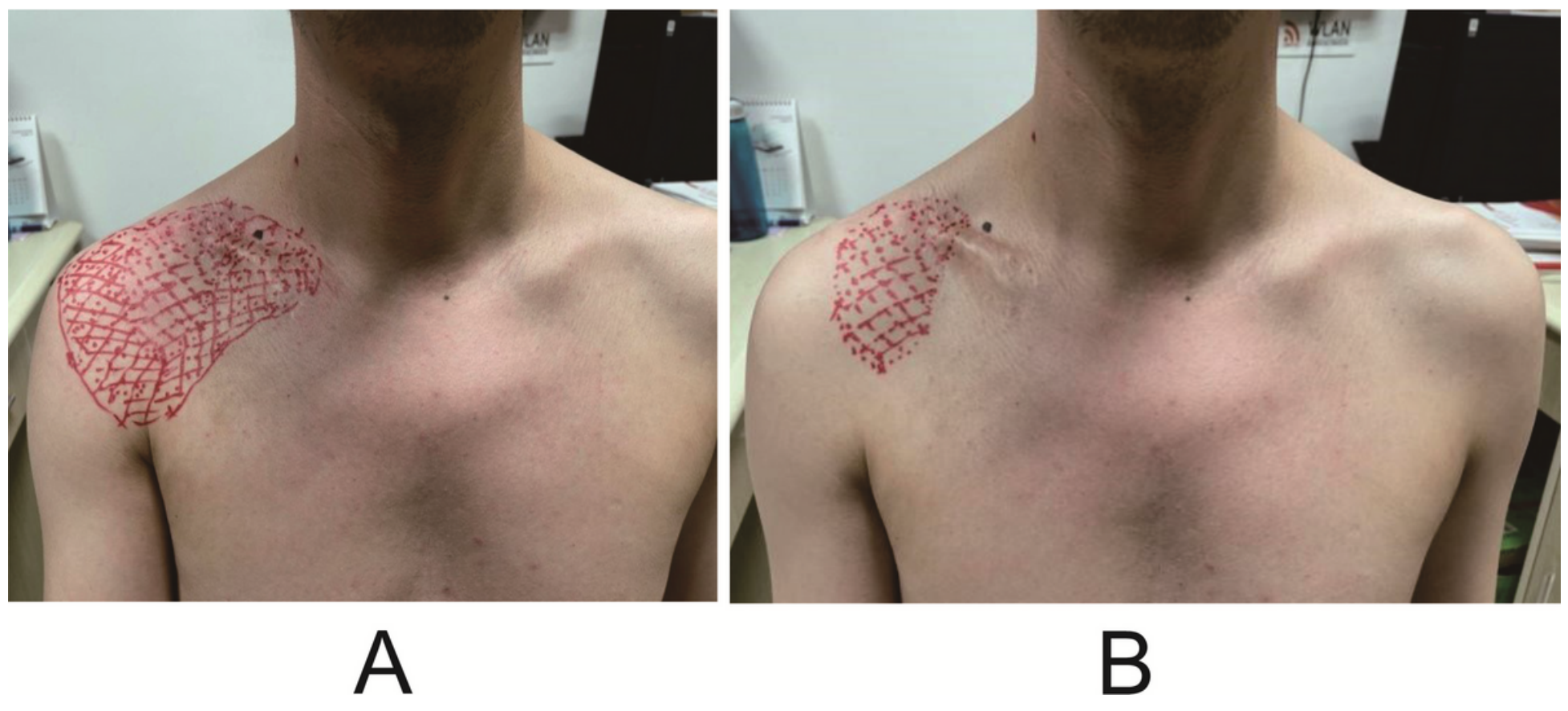

\section{$\mathrm{B}$}

Figure 3

A 32-year-old male injured supraclavicular nerve during the second incision for removing the internal fixation, and suffered from night pain manifested as dull pain and prickling accompanied by itching. $a, b$ The hyperesthesia or hypoesthesia area 